\title{
Depth inversion in shallow water based on nonlinear properties of shoaling periodic waves
}

\author{
Stéphan T. Grilli \\ Department of Ocean Engineering, University of Rhode Island, Narragansett, RI 02882, USA
}

Received 20 November 1997; revised 26 May 1998; accepted 25 June 1998

\begin{abstract}
Two depth inversion algorithms (DIA) applicable to coastal waters are developed, calibrated, and validated based on results of computations of periodic waves shoaling over mild slopes, in a two-dimensional numerical wave tank based on fully nonlinear potential flow (FNPF) theory. In actual field situations, these algorithms would be used to predict the cross-shore depth variation $h$ based on sets of values of wave celerity $c$ and length $L$, and either wave height $H$ or left-right asymmetry $s_{2} / s_{1}$, simultaneously measured at a number of locations in the direction of wave propagation, e.g., using video or radar remote sensing techniques. In these DIAs, an empirical relationship, calibrated for a series of computations in the numerical wave tank, is used to express $c$ as a function of relative depth $k_{\mathrm{o}} h$ and deep water steepness $k_{\mathrm{o}} H_{\mathrm{o}}$. To carry out depth inversion, wave period is first predicted as the mean of observed $L / c$ values, and $H_{\mathrm{o}}$ is then predicted, either based on observed $H$ or $s_{2} / s_{1}$ values. The celerity relationship is finally inverted to predict depth $h$. The algorithms are validated by applying them to results of computations for cases with more complex bottom topography and different incident waves than in the original calibration computations. In all cases, root-mean-square ( $\mathrm{rms}$ )-errors for the depth predictions are found to be less than a few percent, whereas depth predictions based on the linear dispersion relationship-which is still the basis for many state-of-the-art DIAs-have rms-errors 5 to 10 times larger. (C) 1998 Elsevier Science B.V. All rights reserved.
\end{abstract}

Keywords: Nonlinear surface waves; Wave shoaling; Inverse problem; Nearshore topography; Boundary element method; Coastal engineering

\footnotetext{
*Fax: + 1-401-874-6837; E-mail: grilli@ mistral.oce.uri.edu
} 


\section{Introduction}

Detailed knowledge of the ocean bottom topography is of great importance in many coastal engineering problems. In shallow water, more particularly, due to active sediment transport, the bottom topography is subjected to large changes on various time scales and it is thus desirable for a variety of applications to develop methods allowing for easy and continuous monitoring of topographical changes. The present study deals with a class of depth prediction methods, developed for coastal areas, which use characteristics of shoaling waves measured on the ocean surface to predict the bottom topography; such methods are referred to as depth inversion algorithms (DIAs).

Real ocean waves propagating toward a shore usually appear to be multi-frequency and multi-directional (their frequency-direction distribution of energy can be represented using a directional wave spectrum) with, often, a leading (or spectral peak) wave frequency and a main direction of propagation generally oblique to the coast, with some directional spreading. Coastal currents resulting from the combined effects of tides, wind, local estuaries, and meso-scale oceanic circulation may also exist and influence wave propagation. Although more complex cases will be addressed in future work following the same methodology, for demonstrating the proposed DIAs, the present study will be limited to periodic waves propagating/shoaling in a direction normal to the shore. The coastal topography will be assumed to be constant in the along-shore direction and mildly sloping in the cross-shore direction. Hence, this study will essentially be a two-dimensional one in a vertical plane. It will also be assumed that there are no significant mean currents, except for those induced by waves in the cross-shore direction (mean mass transport, undertow).

In the coastal region, measurements of shoaling wave characteristics have been made using shore-based video techniques, usually following the methodology introduced by Lippmann and Holman (1989, 1990), or longer range remote sensing techniques (radar, electro-optical), such as in the work of Dugan et al. (1996), Dugan (1997) and Williams and Dugan (1997). In the DIAs used to process the data obtained with such methods, the nearshore bathymetry is retrieved using measured wave celerities, by inverting a wave-celerity-to-depth relationship obtained from a wave theory (i.e., a dispersion relationship). In radar remote sensing techniques, measurements are made for the modulations of the slope of scatterers dispersed on the ocean surface (e.g., capillary waves), due to longer propagating gravity waves. Assuming a long-crested periodic swell shoaling-up towards a long straight coast, spatial wave phase variations in the direction $x$ normal to the shore can be retrieved from the scatterers' slope modulations, using a relevant modulation transfer function (Dugan, 1997; Williams and Dugan, 1997). In video techniques, wave phases are similarly estimated based on variations of average surface brightness obtained in video time exposures (Lippmann and Holman, 1989, 1990, 1991, 1992). Phases, in turn, provide spatial variations of wavelength $L(x)$ and, when multiple 'snapshots' of the ocean surface are used over a short period of time, ensuring a quasi-steady wave field (typically several tens of seconds for airborne remotely sensed data), phase celerity variations can be obtained as, $c(x) \simeq \Delta x_{\mathrm{p}} / \Delta t$ (with $\Delta x_{\mathrm{p}}$, the distance between two successive locations of a line of constant phase in the snapshots, and $\Delta t$ the time interval between snapshots). From the $L$ and $c$ data, 
wave period can be estimated as, $T \simeq \overline{L / c}$ (where the overline indicates the mean of measured values). The bottom depth variation $h(x)$ is finally obtained from an inverted dispersion relationship, relating $c$ and $h$, for a given $T$.

In shoaling waves, particularly in shallow water when approaching breaking, nonlinearity continuously increases and strongly influences wave celerity, resulting in wellknown amplitude dispersion effects, which lead to larger propagation speeds for higher waves (e.g., Svendsen and Jonsson, 1980; Dean and Dalrymple, 1984). In state-of-the-art DIAs used in remote sensing, the linear dispersion relationship (Dean and Dalrymple, 1984) is often used to perform depth inversion (Dugan, 1997; Williams and Dugan, 1997); hence, no amplitude dispersion effects are included and frequency dispersion is only included to first-order. In DIAs used in combination with video techniques, which are mostly applied to nearshore regions and to the surf-zone, wave celerity is typically based on the nonlinear shallow water wave equations (NSWE), i.e., $c=\sqrt{g(h+H)}$, with $H$ the wave height (Lippmann and Holman, 1989, 1990, 1991); hence, amplitude dispersion effects are included to first-order in nonlinearity, but there is no frequency dispersion. No theoretical relationship exists that exactly predicts the celerity of fully nonlinear shoaling waves. Numerical computations, however, can be used to do so. Using a model solving fully nonlinear potential flow (FNPF) equations, i.e., a numerical wave tank, Grilli and Horrillo (1996, 1997b) computed detailed characteristics of periodic waves shoaling over mild slopes. This model is both numerically exact and experimentally validated up to and slightly beyond the breaking point. Results showed, for various incident waves, both significant frequency and amplitude dispersion effects on wave celerity, up to the breaking point, as well as effects due to increasing wave left to right asymmetry. The results also showed that the celerity variations predicted by linear, cubic, or even higher-order Stokes theories failed to capture the correct celerity variation, particularly, closer to the breaking point. Using a similar model, Grilli et al. (1994) showed that the NSWE failed, even for long waves such as solitary waves, to correctly predict the celerity variation, when approaching breaking. This is in part because of the increasing left to right asymmetry in the waves, as they shoal-up the slope, and in part because of the lack of dispersive effects in the equation. The latter was confirmed by the computations of Wei et al. (1995), who used a long wave Boussinesq model including both linear dispersive and fully nonlinear effects, and showed a better agreement with FNPF results for long waves than the NSWE.

As we will see, significant errors in depth prediction can result from neglecting or not correctly accounting for both amplitude and frequency dispersion effects on wave celerity in DIAs. If celerity corrections due to wave nonlinearity are to be calculated, however, simultaneous wave height information $H(x)$ is also required. In the depth prediction methods based on video techniques, wave elevation can be obtained through correlation with variations of surface brightness (Lippmann and Holman, 1991). Wave height information $H(x)$ can also be obtained using radar altimetry, based on traveling times of radar waves. Accurate height measurements based on remote sensing techniques such as synthetic aperture radar, however, are still quite problematic under the current state of the technology.

In the present study, results of the fully nonlinear computations by Grilli and Horrillo (1996, 1997b) wave shoaling in a numerical wave tank are used to develop, calibrate, 
and test two new DIAs which could be used to predict depth in coastal areas, in combination with measurements of surface waves obtained, e.g., from video or radar remote sensing techniques. In the first algorithm (referred to as DIA1), it is assumed that a data set of simultaneous $(c, L, H)$ values is available for a series of $x$ locations in the cross-shore direction. After validating and testing DIA1, a second algorithm (referred to as DIA2) is proposed which eliminates the use of $H$ and only relies on phase informations. The new DIAs are validated by predicting the bottom topography, using wave shoaling characteristics computed in the numerical wave tank, for more complex cases than in the original calibration computations.

It should be pointed out that, in addition to the restriction to normally incident periodic waves, there are many other uncertainties in this problem, not considered here, pertaining to the generation of the data set itself using, video, remote sensing, or other techniques. These uncertainties will affect the accuracy of the depth prediction using the present DIAs, the same way as when using other existing DIAs.

\section{Periodic wave shoaling and the depth inversion problem}

Periodic progressive waves in intermediate and shallow water undergo transformations which, according to classical wave theories (Dean and Dalrymple, 1984), are
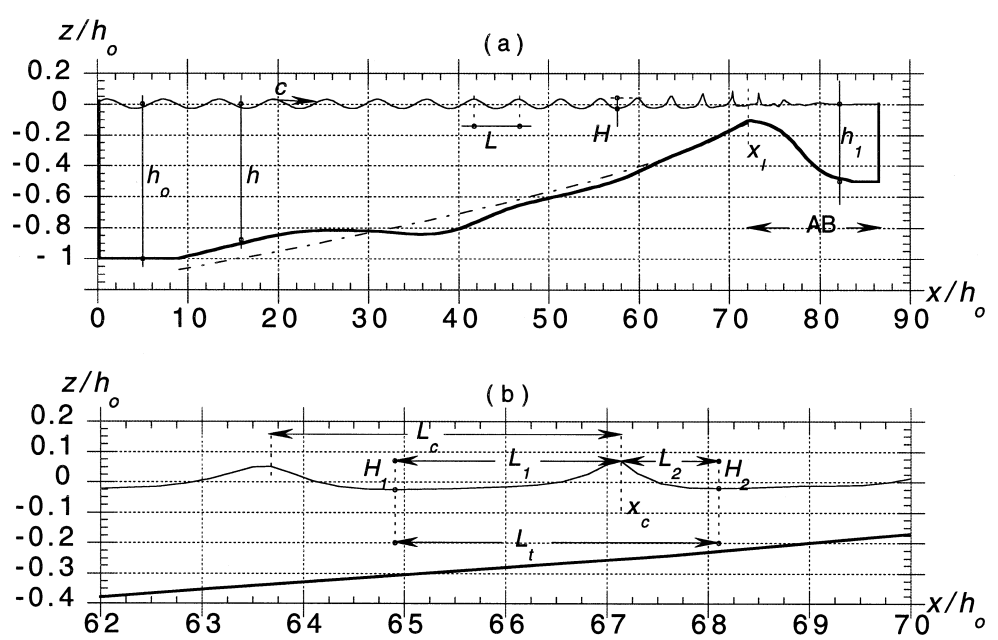

Fig. 1. (a) Typical sketch for periodic wave shoaling computations in the numerical wave tank. The bottom topography (—) corresponds to the average of five depth transects taken at Ft. Walton Beach, FL on Sept. 29, 1994, with $h_{\mathrm{o}}=18.53 \mathrm{~m}$ and an average slope $1: 82$; and (_- - ) is the least square fit to the topography: $h / h_{\mathrm{o}}=0.0758\left(74.20-x / h_{\mathrm{o}}\right)^{0.64}$ (with $R^{2}=0.98$ ). (b) Blow-up of (a) and definition of some of the wave geometric characteristics used in DIA1 and DIA2. 
function of their deep water characteristics, namely height $H_{\mathrm{o}}$ and period $T$, and of the ocean bottom topography, i.e., depth $h$. For idealized two-dimensional problems in a vertical plane normal to the shore (Fig. 1), these transformations, referred to as wave shoaling, are usually expressed as the variation of wave celerity $c$, or length $L=c T$, and height $H$, as a function of the distance $x$ measured from a deep water depth limit or from the shore. To the first-order in wave steepness $k H(k=2 \pi / L$ being the wave number) and in the absence of a mean current, linear Stokes theory predicts wave celerity $c_{l}$ as a function of depth as (e.g., Dean and Dalrymple, 1984; note, an index $l$ will be used from now on to indicate values predicted by linear wave theory),

$$
c_{l}=c_{\mathrm{o}} \tanh k h ; \text { with } k=\frac{2 \pi}{T c_{l}}, c_{o}=\frac{g T}{2 \pi},
$$

the deep water linear wave celerity, and $g$ the acceleration of gravity. Eq. (1) is referred to as the linear frequency dispersion relationship and predicts a decrease in wave celerity with decreasing $T$ or $h$. By inverting this equation for a given $T$, it is possible to estimate the depth variation $h(x)$ in the direction $x$ of wave propagation, based on $c(x)$ values measured at the free surface. This simple method is in fact the basis for most state-of-the-art DIAs used in combination with remotely sensed field data to estimate the ocean bottom topography in coastal areas (Dugan, 1997; Williams and Dugan, 1997).

No effects of wave height are included in Eq. (1). These, according to Stokes theory, only show up at third-order in $\mathrm{kH}$ as so-called amplitude dispersion effects; we thus get, for the third-order celerity (e.g., Svendsen and Jonsson, 1980),

$$
c_{3}=c_{l}\left[1+(k H)^{2} \mathscr{F}_{l}(k h)\right] ; \text { with } \mathscr{F}_{l}=\frac{8+\cosh 4 k h-2 \tanh ^{2} k h}{32 \sinh ^{4} k h},
$$

a monotonously increasing function for decreasing relative depth $k h$. A simple prediction of amplitude dispersion effects can be made with Eq. (2), by estimating the variation of wave steepness as a function of both $k h$ and the deep water steepness $k_{\mathrm{o}} H_{\mathrm{o}}$, using linear wave theory (where, $k_{\mathrm{o}}=2 \pi / L_{\mathrm{o}} ; L_{\mathrm{o}}=c_{\mathrm{o}} T$ ); this is found as,

$$
k_{l} H_{l}=k_{\mathrm{o}} H_{\mathrm{o}} \mathscr{G}_{l}(k h) ; \text { with } \mathscr{G}_{l}=(\tanh k h)^{-3 / 2}\left[1+\frac{2 k h}{\sinh 2 k h}\right]^{-1 / 2} \text {, }
$$

a monotonously increasing function, when $k h$ decreases (Dean and Dalrymple, 1984). The ratio $c_{3} / c_{l}$ can thus be calculated and we see, for instance, that for a weakly nonlinear incident wave with $k_{\mathrm{o}} H_{\mathrm{o}}=0.01$, the increase in celerity with respect to linear wave theory reaches $0.07 \%$ for $k h=\pi / 5$ and $5.9 \%$ for $k h=\pi / 10$, the usual shallow water depth limit (Dean and Dalrymple, 1984); for a mildly nonlinear incident wave with $k_{\mathrm{o}} H_{\mathrm{o}}=0.05$, these increases become $1.7 \%$ and $246 \%$, respectively. (Note, the third-order dispersion Eq. (2) diverges in shallower water and/or for strong wave nonlinearity; actual shoaling waves also do not keep the permanent symmetric shape assumed in Stokes theory.) Hence, for sufficiently nonlinear waves and shallow water, due to amplitude dispersion effects, linear wave theory may greatly underestimate wave celerity, leading to poor depth prediction with DIAs based on Eq. (1), particularly in 
areas where depth is the more subject to change and thus depth information is the most needed. This is well known and was recently illustrated by Dugan (1997) and Williams and Dugan (1997), in the analysis of their field measurements using a DIA based on linear wave theory.

The above analysis is rather approximate but, nevertheless, gives the key indication that, as waves shoal-up over a sloping bottom and become both higher and steeper, amplitude dispersion effects on wave celerity cannot be neglected in the depth inversion problem. Such effects alone, however, are not sufficient to predict celerity and, as discussed in the introduction, the celerity of (non-dispersive) NSWE fails, even for long shoaling waves, to accurately predict the correct celerity variation. For instance, for solitary waves of height initially 0.2 and 0.4 times the depth, shoaling over a 1:35 slope, Grilli et al. (1994) reported maximum errors of $52 \%$ and $24 \%$, respectively, on the wave celerity up to the breaking point, for the prediction of NSWE as compared to FNPF results.

More accurate celerity variations over complex bottom topographies can be obtained using numerical models which include both frequency and amplitude dispersion effects. Many numerical studies have dealt with the modeling of wave shoaling over an arbitrary bottom topography, i.e., the direct problem, using models based, e.g., on weakly nonlinear Boussinesq equations with modified or extended linear dispersion properties (Madsen et al., 1991; Nwogu, 1993), and/or extended nonlinear properties (Wei et al., 1995), or on fully nonlinear steady wave theories (e.g., Sobey and Bando, 1991). Linear dispersion properties of shoaling waves are correctly represented in modified Boussinesq models, but nonlinear properties are only included up to limited order, depending on the truncation error of the series used to represent free surface boundary conditions (Wei et al., 1995). Hence, despite their satisfactory predictions for a wide range of water depth, Boussinesq models may not be sufficiently accurate to predict wave shape and kinematics in very shallow water and close to breaking, where nonlinearity is strong and wave height is on the order of water depth. Quasi-steady models based on higher-order wave theories, such as the Fourier steady wave theory (Sobey and Bando, 1991), which lack the key influence of wave left-right asymmetry, also do not provide accurate predictions of shoaling wave characteristics in shallow water, where waves take a forward tilted shape (Grilli and Horrillo, 1996, 1997b; see also Fig. 1b). Recent advances in fully nonlinear models based on potential flow theory, i.e., so-called numerical wave tanks (see the work of Tsai and Yue (1996) for a review), have made it possible to calculate 'numerically exact' properties of shoaling waves, up to the breaking point, to within a few percent of laboratory measurements (Grilli et al., 1994; Ohyama et al., 1994; Grilli and Horrillo, 1996; Grilli et al., 1997; Grilli and Horrillo, 1997a; Grilli and Horrillo, 1997b). FNPF equations, in fact, are the governing equations from which most approximate wave theories or models (such as Boussinesq equations) are derived, and are thus able to provide an accurate representation of surface waves, independent of depth or nonlinearity parameters such as $k h$ or $k H$. The only limitation is that friction and vorticity must be negligible, which is a rather good approximation before waves break (Dommermuth et al., 1988). In numerical wave tanks, mechanisms must be provided for wave generation at an offshore boundary and wave energy absorption at the shore, before breaking occurs (Fig. 1a). More details are given in the Section 3. 
Thus, for periodic waves shoaling over mild slopes, Grilli and Horrillo (1996, 1997b) used the FNPF numerical wave tank by Grilli and Subramanya (1996), with the wave generation and absorption methods developed by Grilli and Horrillo (1997a), to calculate celerity and height variations for many different incident waves shoaling over mild slopes ranging from 1:35 to 1:70. They showed that, although a slope is needed in the tank to induce the necessary left-right asymmetry in shoaling waves, for a mild slope, the variations of $c$ and $H$ do not depend very much on the magnitude of the slope, provided they are compared for the same normalized depth $k_{\mathrm{o}} h$ or $k h$. Full nonlinearity, however, is important in shallow water and large differences (up to $80 \%$ for $c$ and $H$ ) may occur between predictions of linear or weakly nonlinear wave theories, and the fully nonlinear results. In the present study, Grilli and Horrillo's computations for periodic waves shoaling over a 1:50 plane slope (i.e., in the middle of the tested range of slopes) are used to develop and calibrate two new DIAs for coastal waters.

\section{Review of FNPF numerical wave tank}

\subsection{Governing equations and boundary conditions}

A typical domain for wave shoaling computations is sketched in Fig. 1a. Waves are long-crested and propagate in the $x$ direction normal to the shore. FNPF equations are solved in the numerical wave tank, defined by the free surface, the bottom, a leftward wavemaking boundary and a rightward absorbing boundary. These equations are the governing continuity equation, a no-flow condition on the bottom, nonlinear kinematic and dynamic conditions on the free surface and specified velocity and accelerations on the leftward and rightward boundaries (see the works of Grilli and Subramanya (1996) and Grilli and Horrillo (1997a) for details).

Although this numerical wave tank is general, incident waves in the present study have been limited to periodic progressive waves. Following the work of Grilli and Horrillo (1997a), in order to get permanent form finite amplitude incident waves, so-called streamfunction waves (Dean and Dalrymple, 1984) are generated on the model leftward boundary. These waves, unlike finite amplitude waves produced by a wavemaker, do not exhibit the generation of higher-order harmonics and the beat phenomenon observed in wave tanks, as they propagate over constant depth. Since nonlinear waves have a nonzero mass flux, to ensure constant volume in the tank, incident streamfunction waves are generated together with a mean current, equal and opposite to their period-averaged mass transport velocity. For an actual beach, this situation would correspond to specifying a vertical boundary at some distance from the shore, for which the undertow current balances the incident mass flux. Note that, despite this offshore zero-mass-flux condition, a mean flow circulation occurs within the tank, as it does on a beach, i.e., with an onshore mean mass transport above wave troughs and an offshore mean mass transport below wave troughs; mean-water-level set-down also occurs in the tank (Dean and Dalrymple, 1984; Svendsen and Jonsson, 1980).

In a FNPF numerical wave tank, computations can model overturning waves but, by nature, are limited to the time prior to that at which touch-down of a breaker jet first 
occurs. This does not pose problems when solitary waves are used in the analysis (Grilli et al., 1994, 1997). For periodic waves, however, an absorbing beach must be used to dissipate the energy of each successive incident wave, hence both eliminating reflection and preventing waves from breaking at the top of the slope, while letting them shoal up to very close to breaking. Here, the absorbing beach is specified over a shallower region in the upper part of the slope, whose geometry is assumed somewhat similar to a natural bar on a beach (Fig. 1a). Energy absorption combines both free surface and lateral absorption, with an adaptive calibration of the absorption coefficient (Grilli and Horrillo, 1997a).

(i) An exterior counteracting pressure is specified on the absorbing beach free surface, as proportional to the normal particle velocity, to create a negative work against incident waves; this method can be shown to absorb high frequency wave energy well.

(ii) An active piston-like absorbing boundary condition is specified at the tank rightward extremity; this can be shown to absorb low frequency wave energy well.

(iii) The absorption coefficient in the beach is adaptively calibrated in time to absorb the period-averaged energy of incident waves entering the beach at $x=x_{l}$.

Grilli and Horrillo showed that, when using the above methods, reflection from the beach can be reduced to a few percent. Reflection from the sloping bottom, however, still occurs as it does in nature, but is rather small for mild slopes.

In the results, when not otherwise mentioned, primes indicate nondimensional variables in which lengths have been divided by $h_{\mathrm{o}}$, a reference depth, and times by $\sqrt{h_{\mathrm{o}} / g}$, a characteristic time.

\subsection{Numerical methods}

The continuity equation is solved using a higher-order boundary element method (BEM) based on Green's 2nd identity (Grilli et al., 1989). Boundaries are discretized using nodes and higher-order elements are specified to interpolate in between the nodes. Quadratic elements are used on lateral and bottom boundaries and cubic elements ensuring continuity of the slope are used on the free surface boundary (Grilli and Subramanya, 1996). Fully nonlinear kinematic and dynamic boundary conditions are used on the free surface without any approximation. Time stepping of the geometry and boundary conditions is based on second-order Taylor series expansions, expressed in terms of a time step and of the Lagrangian time derivative. Numerical errors are kept to a very small value by adaptively selecting the time step based on a mesh Courant number. In shoaling computations, as waves become increasingly steep towards the top of the slope, discretization nodes may get too close to each other and create almost singular values for the BEM integrals, leading to poor accuracy. To prevent this, an adaptive regridding method is used to automatically regrid nodes three by three when the distance between two nodes is either more than 4 times or less than 0.25 times the distance between the previous two nodes. In the present applications, a minimum of 20 nodes per wavelength has been maintained on the free surface throughout shoaling computations.

Details of model equations, numerical methods, and validation applications can be found in the above-referenced papers. In the present study, numerical parameters have been selected such as to ensure optimal accuracy of computations. 


\subsection{Computation of shoaling wave properties}

In computations of periodic wave shoaling, the numerical wave tank is assumed to be initially at rest (cold start). At the start of computations, incident periodic waves are generated in the tank by specifying their shape and kinematics along the leftward wavemaking boundary (a tapering function is used over the first three periods). For zero-mass-flux streamfunction waves, kinematics is obtained as a function of initial wave height $H_{\mathrm{o}}$, period $T$, and initial depth $h_{\mathrm{o}}$. When starting computations in deep water (i.e., for $h_{\mathrm{o}} \geq L_{\mathrm{o}} / 2$ ), it was observed that small incident periodic waves closely followed results of linear wave theory, up to a relative depth $k_{\mathrm{o}} h \simeq 0.4-0.5$. Hence, to limit the extension of the computational domain, computations were initiated at a depth $h_{\mathrm{o}}^{*}$ corresponding to only a fraction of the deep water depth (in the present case $h_{\mathrm{o}}^{*}=0.6 h_{\mathrm{o}}$ ), with a height $H_{\mathrm{o}}^{*}$. The corresponding deep water wave height $H_{\mathrm{o}}$ was calculated using the linear wave shoaling coefficient, $K_{\mathrm{s} l}=\left(c_{l} / c_{\mathrm{o}}\right) \mathscr{G}_{l}(k h)$, as $H_{\mathrm{o}}=$ $H_{\mathrm{o}}^{*} / K_{\mathrm{s} l}\left(h_{\mathrm{o}}^{*}\right)$.

Table 1 gives a summary of input parameters for the nine waves used in the calibration of the DIAs and Table 2, for five DIAs' validation applications. All of the initial waves in Table 1 and most of the waves in Table 2 are specified with $H_{\mathrm{o}} *<H_{\mathrm{o}}$, i.e., in the region of initial decrease in wave height which occurs during shoaling (as predicted by linear wave theory).

In computations, each crest and trough of the shoaling wave train are independently identified and followed in space and time, from their generation at the wavemaking boundary to their absorption in the absorbing beach. From these results, envelopes of crest and trough elevations are calculated for successive waves, and wave height $H(x)$ is thus obtained. Using the same results, the phase velocity of successive waves is calculated as $c(x)=\mathrm{d} x_{\mathrm{c}} / \mathrm{d} t$, where $x_{\mathrm{c}}(t)$ denotes the instantaneous crest location for a given wave (Fig. 1b). The wavelength is calculated two ways from the spatial wave profiles, as the horizontal distance between (Fig. 1b): (i) a crest and the previous one, $L_{\mathrm{c}}(x)$; or (ii) between a trough and the next one, $L_{\mathrm{t}}(x)$. Results will show that, for a given depth (or $x$ location), the mean of $L_{\mathrm{c}}$ and $L_{\mathrm{t}}$ is a good prediction of the

Table 1

\begin{tabular}{llllllll}
\hline Number & $H_{\mathrm{o}}^{\prime}$ & $T^{\prime}$ & $H_{\mathrm{o}}^{\prime *}$ & $k_{\mathrm{o}}^{\prime}$ & $k_{\mathrm{o}} h_{\mathrm{o}}^{*}$ & $k_{\mathrm{o}} H_{\mathrm{o}}$ & $c_{\mathrm{o}}^{\prime}$ \\
\hline 1 & 0.0435 & 5.5 & 0.04 & 1.305 & 0.79 & 0.0568 & 0.8754 \\
2 & 0.0653 & 5.5 & 0.06 & 1.305 & 0.79 & 0.0852 & 0.8754 \\
3 & 0.0871 & 5.5 & 0.08 & 1.305 & 0.79 & 0.1137 & 0.8754 \\
4 & 0.0424 & 6.5 & 0.04 & 0.934 & 0.56 & 0.0396 & 1.0345 \\
5 & 0.0636 & 6.5 & 0.06 & 0.934 & 0.56 & 0.0594 & 1.0345 \\
6 & 0.0848 & 6.5 & 0.08 & 0.934 & 0.56 & 0.0792 & 1.0345 \\
7 & 0.0409 & 7.5 & 0.04 & 0.702 & 0.42 & 0.0287 & 1.1937 \\
8 & 0.0614 & 7.5 & 0.06 & 0.702 & 0.42 & 0.0431 & 1.1937 \\
9 & 0.0819 & 7.5 & 0.08 & 0.702 & 0.42 & 0.0575 & 1.1937 \\
\hline
\end{tabular}

Input characteristics of incident waves in the numerical wave tank for shoaling over a 1:50 slope: $H_{\mathrm{o}}^{\prime}=$ $H_{\mathrm{o}}^{\prime *} / K_{\mathrm{s} l}\left(h_{\mathrm{o}}^{\prime *}\right)$ deep water wave height; $T^{\prime}$ wave period; $H_{\mathrm{o}}^{\prime}$ wave height at initial offshore depth $h_{\mathrm{o}}^{\prime *}=0.6$; $k_{\mathrm{o}}^{\prime}=\left(2 \pi / T^{\prime}\right)^{2}$ and $c_{\mathrm{o}}^{\prime}=T^{\prime} / 2 \pi$ (linear) deep water wave number and celerity, respectively. 
Table 2

Data and global results for five depth inversion cases

\begin{tabular}{llllllllllll}
\hline Case & $H_{\mathrm{o}}^{\prime}$ & \multicolumn{1}{l}{$T^{\prime}$} & $H_{\mathrm{o}}^{\prime *}$ & \multicolumn{1}{c}{$T_{\mathrm{p}}^{\prime}$} & \multicolumn{1}{l}{$H_{\mathrm{po}}^{\prime}$} & $\mathrm{rms}$ & $\varepsilon_{\mathrm{m}}$ & $\mathrm{rms}_{l}$ & $\varepsilon_{l \mathrm{~m}}$ & $\mathrm{rms}_{c}$ & $\mathrm{rms}_{H}$ \\
\hline $\mathrm{b} 1$ & 0.0352 & 11.47 & 0.04 & 11.55 & 0.0349 & 3.6 & 17.2 & 15.6 & 47.6 & 1.2 & 2.8 \\
$\mathrm{~b} 2$ & 0.0352 & 11.47 & 0.04 & 11.50 & 0.0350 & 2.7 & 8.6 & 16.2 & 50.8 & 1.1 & 3.8 \\
$\mathrm{n} 1$ & 0.0653 & 5.5 & 0.06 & 5.52 & 0.0646 & 2.1 & 8.0 & 14.6 & 50.3 & 0.6 & 2.7 \\
$\mathrm{n} 2$ & 0.0636 & 6.5 & 0.06 & 6.52 & 0.0625 & 1.7 & 7.1 & 17.0 & 57.6 & 0.5 & 2.0 \\
$\mathrm{n} 3$ & 0.0614 & 7.5 & 0.06 & 7.51 & 0.0602 & 2.0 & 13.9 & 20.5 & 67.9 & 0.4 & 2.0 \\
\hline
\end{tabular}

Input characteristics of incident waves in the numerical wave tank for both DIAs: $H_{\mathrm{o}}^{\prime}$ deep water wave height; $T^{\prime}$ wave period; $H_{\mathrm{o}}^{\prime *}$ wave height specified at initial offshore depth, $h_{\mathrm{o}}^{\prime *}=0.6$. Note $k_{\mathrm{o}} H_{\mathrm{o}}=0.0105$ for cases b1 and b2 and can be found in Table 1 for cases n1-n3.

Predicted wave characteristics in DIA1: $T_{\mathrm{p}}^{\prime}$ wave period; $H_{\mathrm{po}}^{\prime}$ deep water wave height; $\left(\mathrm{rms}, \varepsilon_{\mathrm{m}}\right)$, rms and maximum relative errors $(\%)$ for depth prediction with DIA1; $\left(\mathrm{rms}_{l}, \varepsilon_{l \mathrm{~m}}\right)$, rms and maximum relative errors $(\%)$ for depth prediction with the linear dispersion relation; $\left(\mathrm{rms}_{c}, \mathrm{rms}_{H}\right)$ rms-errors for direct celerity and depth predictions, in Fig. 9 and Fig. 11.

(unknown) 'true' wavelength $L(x)=c(x) T$, needed to predict the wave period in the DIAs, based on the wave celerity (see Section 4).

After the initial transient wave front has been absorbed in the absorbing beach, results show that values of $H, c$, and $L$ are well reproduced for successive incident waves. Hence, this indicates that computations have reached a quasi-steady state for which reflection, from the slope and from the absorbing beach, is insignificant. To eliminate the small remaining differences between successive waves, in the present applications, wave properties have been averaged over four to six successive waves.

\section{Depth inversion algorithms}

\subsection{Analysis of celerity and height variations for periodic waves}

Results of the computations of Grilli and Horrillo (1996, 1997b) for the nine cases in Table 1 are given in Fig. 2 for the phase celerity normalized by the (linear) deep water celerity $c / c_{\mathrm{o}}$, and in Fig. 3 for the shoaling coefficient $K_{\mathrm{s}}=H / H_{\mathrm{o}}$, both as a function of normalized depth $k_{\mathrm{o}} h$.

In Fig. 2, as expected from earlier discussions, for $k_{\mathrm{o}} h<0.5$, the shallower the normalized depth and the larger the incident wave steepness $k_{\mathrm{o}} H_{\mathrm{o}}$ (i.e., the larger the incident wave height and the smaller the period), the larger the increase of the nonlinear wave celerity with respect to $c_{l}$. As discussed above, such celerity increases mostly result from amplitude dispersion effects due to increasing wave steepness $k H$ during shoaling. For the nine computed cases, these effects lead, in shallow water $(k h<\pi / 10)$, to a 40 to $85 \%$ maximum increase in celerity with respect to $c_{l}$. Note that the Ursell number (Svendsen and Jonsson, 1980), $U_{\mathrm{r}}=k H /(k h)^{3}$, used in (weakly nonlinear) long wave theories such as Boussinesq's to express the ratio of nonlinear to dispersive effects, does not collapse the celerity curves in Fig. 2 to a single curve, when used as an abscissa instead of $k_{\mathrm{o}} h$. This indicates that a single parameter such as $U_{\mathrm{r}}$ is not 


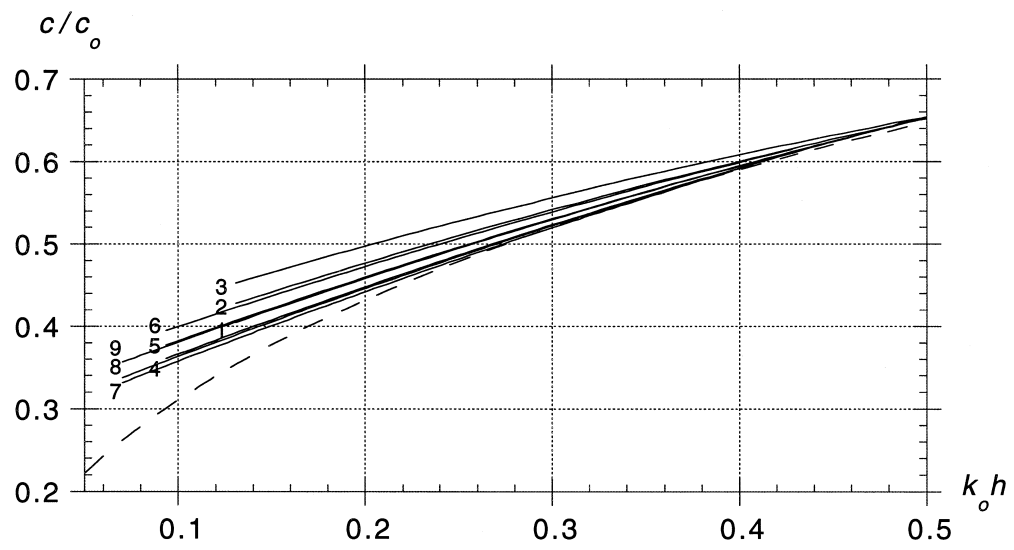

Fig. 2. Wave celerity $c / c_{\mathrm{o}}$ computed in the numerical wave tank over a 1:50 slope, for the nine cases listed in Table 1. (---) $c_{l} / c_{\mathrm{o}}=\tanh k h$, linear wave celerity.

sufficient to describe strongly nonlinear and dispersive effects observed in shoaling waves. In Fig. 3, a similar analysis is presented for the nonlinear shoaling coefficient, and we see that linear wave theory also significantly underpredicts wave height in the same depth region where celerity is underpredicted in Fig. 2; for the nine studied cases the underprediction of $K_{\mathrm{s}}$ by $K_{\mathrm{s} l}$ reaches up to $55 \%$.

In view of these results, Grilli and Horrillo $(1996,1997 b)$ computed the quantity $K_{\mathrm{s}} /\left(c / c_{\mathrm{o}}\right)=k H /\left(k_{\mathrm{o}} H_{\mathrm{o}}\right)$ (i.e., the local wave steepness normalized by the deep water steepness) and, due to the partial compensation of underpredictions of wave height and celerity, observed that this ratio showed much less variations with respect to the linear wave result, $k_{l} H_{l} /\left(k_{\mathrm{o}} H_{\mathrm{o}}\right)=\mathscr{G}_{l}$ (see Eq. (3)), than its numerator and denominator taken

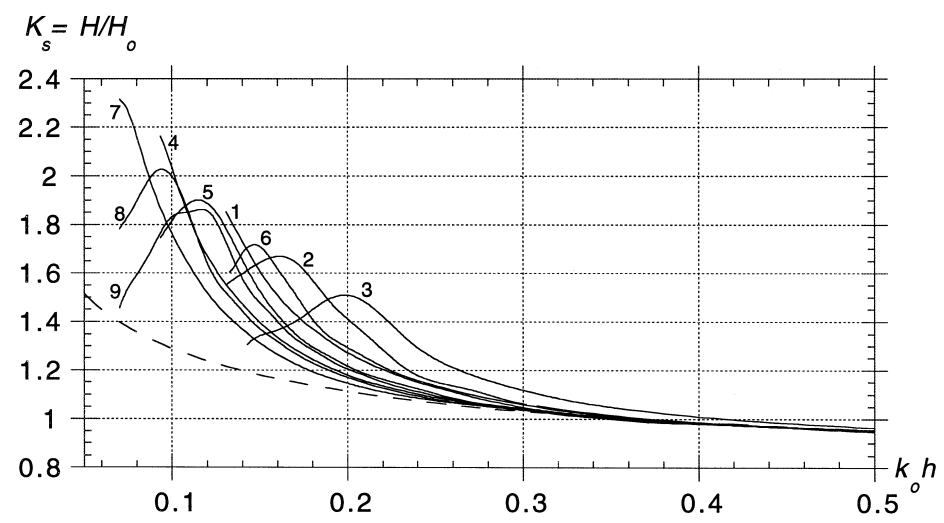

Fig. 3. Wave height $H / H_{\mathrm{o}}$ computed in the numerical wave tank over a 1:50 slope, for the nine cases listed in Table 1. (-- ) $K_{\mathrm{s} l}=\left(c_{l} / c_{\mathrm{o}}\right) \mathscr{G}_{l}(k h)$, linear wave shoaling coefficient. 
independently. Fig. 4 shows a comparison of normalized nonlinear steepnesses $k H / k_{\mathrm{o}} H_{\mathrm{o}}=H /\left(H_{\mathrm{o}} c / c_{\mathrm{o}}\right)$, calculated for the cases in Table 1 , with $\mathscr{G}_{l}$. The latter is found to explain at least $98 \%$ of the variance of the numerical results. This rather unexpected result will be used as a basis for predicting $H_{\mathrm{o}}$, from observed values of $H$ and $c$, in one of the DIAs presented in Section 4.2 (DIA1).

To be able to express the nonlinear celerity variation for other values of the two parameters $k_{\mathrm{o}} H_{\mathrm{o}}$ and $k_{\mathrm{o}} h$ than for the cases in Table 1 , and considering the low sensitivity of results to detailed bottom shape (provided the slope is mild), an empirical function was fitted to the celerities computed in Fig. 2, in the depth region where discrepancies with linear wave theory are significant $\left(k_{\mathrm{o}} h \leq 0.5\right)$. A bi-quadratic function of the two parameters was found to give good results.

$$
\frac{c}{c_{\mathrm{o}}}=A_{0}+A_{1}\left(k_{\mathrm{o}} h\right)+A_{2}\left(k_{\mathrm{o}} H_{\mathrm{o}}\right)+A_{3}\left(k_{\mathrm{o}} h\right)\left(k_{\mathrm{o}} H_{\mathrm{o}}\right)+A_{4}\left(k_{\mathrm{o}} h\right)^{2}+A_{5}\left(k_{\mathrm{o}} H_{\mathrm{o}}\right)^{2}
$$

Values of coefficients $A_{0}$ to $A_{5}$ where found with a least square method, using about 100 multivariate data points per wave case in Table 1 , as $A_{0}=0.2397, A_{1}=1.0063$, $A_{2}=0.9118, A_{3}=-2.2405, A_{4}=-0.3299$, and $A_{5}=1.3292$. The coefficient of determination for this curve fit was $R^{2}=99.8 \%$. Fig. 5 shows $c / c_{\mathrm{o}}$ variations calculated based on Eq. (4), for $k_{\mathrm{o}} h=0$ to 0.5 and $k_{\mathrm{o}} H_{\mathrm{o}}=0.02$ to 0.14 by steps of 0.02 , which approximately covers the range of variation of $k_{\mathrm{o}} H_{\mathrm{o}}$ in Table 1 , where an accurate prediction of celerity can be expected.

In Fig. 5 , the nonlinear celerity curve a $\left(k_{\mathrm{o}} H_{\mathrm{o}}=0.02\right.$, i.e., for the smaller incident steepness) agrees well with linear wave theory, down to depth $k_{\mathrm{o}} h=0.2$. For shallower depth and smaller incident steepness, however, no numerical results are available in Table 1. To be able to cover this parametric region in DIAs, we assumed that the

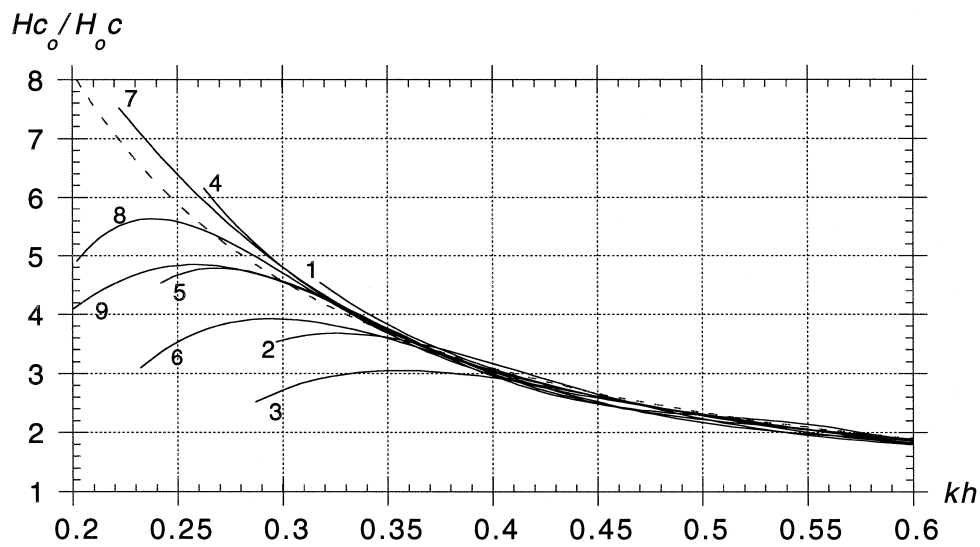

Fig. 4. Normalized wave steepness $k H / k_{\mathrm{o}} H_{\mathrm{o}}=H c_{\mathrm{o}} / H_{\mathrm{o}} c$ computed in the numerical wave tank for the nine cases listed in Table 1, using results of Fig. 2 and Fig. 3. Linear theory gives: (---) $\mathscr{G}_{l}(\mathrm{kh})(\mathrm{Eq}$. (3)). 


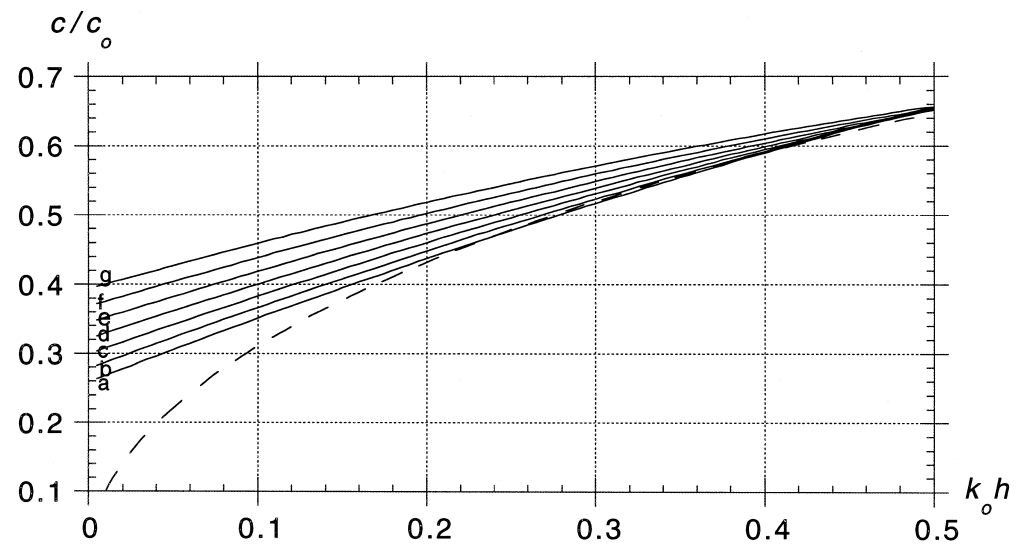

Fig. 5. Empirical curve fit, based on Eq. (4), of wave celerities $c / c_{\mathrm{o}}$ computed in numerical wave tank for the cases in Table 1. Curves a to $g$ correspond to $k_{\mathrm{o}} H_{\mathrm{o}}=0.02$ to 0.14 by step 0.02 . (---) $c_{l} / c_{\mathrm{o}}$, linear wave celerity.

celerity could be found by linear interpolation between results in Fig. 5 for curve a (or a nearby curve) and linear wave theory Eq. (1) as,

$$
\frac{c}{c_{\mathrm{o}}}=\tanh k h+\frac{k_{\mathrm{o}} H_{\mathrm{o}}}{\gamma_{\mathrm{oa}}}\left[\frac{c_{\mathrm{a}}}{c_{\mathrm{o}}}-\tanh k h\right] ; \text { for } k_{\mathrm{o}} H_{\mathrm{o}}<\gamma_{\mathrm{oa}}
$$

where $c_{\mathrm{a}} / c_{\mathrm{o}}$ is the celerity obtained with Eq. (4) for $k_{\mathrm{o}} H_{\mathrm{o}}=\gamma_{\mathrm{oa}}$. Since $k h$ is needed to calculate the tanh function, the following approximation was also made: $k h \simeq$ $k_{\mathrm{o}} h /\left(c_{\mathrm{a}} / c_{\mathrm{o}}\right)$. Eq. (5) was tested in the applications and it was found that the value $\gamma_{\mathrm{oa}}=0.017$ provided better results than the value 0.02 , corresponding to curve a in Fig. 4. Hence, the former value is used in the DIAs.

\subsection{Depth inversion algorithm for (c, H, L) data (DIA1)}

In this depth inversion problem, referred to as DIA1, $N$ sets of values of $c\left(x_{i}\right)$, $H\left(x_{i}\right)$ and $\left(L_{\mathrm{c}}\left(x_{i}\right), L_{\mathrm{t}}\left(x_{i}\right)\right)$ are assumed to be known from simultaneous free surface observations at locations $x_{i}$, for $i=1, \ldots, N$ (e.g., through remote sensing). The problem thus consists in predicting $h\left(x_{i}\right)$, for $i=1, \ldots, N$, based on these observations. Assuming that, for mildly and monotonously sloping bottom, Eq. (4) with the interpolation Eq. (5) (for small incident wave steepness) represent the true nonlinear celerity variation, independent of the details of bottom topography, these equations can be inverted to predict the depth corresponding to a known celerity. As celerity significantly varies with $k_{\mathrm{o}} H_{\mathrm{o}}$, particularly in shallow water (Figs. 2 and 5), both the wave period $T$ (or, $k_{\mathrm{o}}=(2 \pi)^{2} /\left(g T^{2}\right)$ ) and the deep water wave height $H_{\mathrm{o}}$ must be calculated from the data before depth inversion can be performed.

For periodic waves, $T=L / c$ where $L$ is the 'true' wavelength corresponding to the local depth. After several numerical tests, it was found that $L$ could locally be well predicted using the average of $L_{\mathrm{c}}$ and $L_{\mathrm{t}}$ (i.e., the wavelengths measured between two 
successive crests and troughs, respectively; Fig. 1b); thus, in DIA1, $T$ is calculated as the mean of measured $\left[\left(L_{\mathrm{c}}(x)+L_{\mathrm{t}}(x)\right) /(2 c(x))\right]$ values, over the $x_{i}$ locations $(i=$ $1, \ldots, N)$. As indicated before, the wave height $H$ is well predicted by $H_{\mathrm{o}}\left(c / c_{\mathrm{o}}\right) \mathscr{G}_{l}$, with $c / c_{\mathrm{o}}$ the observed nonlinear celerity (Fig. 5). Eq. (3) for $\mathscr{G}_{l}$ can thus be used in DIA1 to estimate $H_{\mathrm{o}}$, when a set of simultaneous values of $H$ and $c$ is available.

Based on the above, the layout for DIA1 is as follows $(i=1, \ldots, N)$.

(1) The period $T$ (and thus $k_{\mathrm{o}}$ ) is first predicted, as explained above, using $L_{\mathrm{c}}\left(x_{i}\right)$, $L_{\mathrm{t}}\left(x_{i}\right)$ and $c\left(x_{i}\right)$ values.

(2) A first guess is made for $H_{\mathrm{o}}$, say $H_{\mathrm{o} 1}$, as the wave height observed at the most offshore location, and a first depth variation $h_{1}\left(x_{i}\right)$ is calculated for this guess (i.e., for $\left.k_{\mathrm{o}} H_{\mathrm{o} 1}\right)$ using the inverted Eqs. (4) and (5) and the $c\left(x_{i}\right)$ values.

(3) Based on $h_{1}\left(x_{i}\right)$, a new corrected value of $H_{\mathrm{o}}$, say $H_{\mathrm{o} 2}$, is obtained by calculating the mean of $\left[H\left(x_{i}\right) /\left(\mathscr{G}_{l}\left(k h_{1}\right)\left(c\left(x_{i}\right) / c_{\mathrm{o}}\right)\right)\right]$ values, with $k h_{1}=k_{\mathrm{o}} h_{1}\left(x_{i}\right) /\left(c\left(x_{i}\right) / c_{\mathrm{o}}\right)$ and Eq. (3) for $\mathscr{G}_{l}$.

(4) Steps (2) and (3) are iteratively repeated, for $n=2,3, \ldots$, until convergence is reached on $H_{\mathrm{o} n}$ and $h_{n}(x)$ according to some preset criteria.

Note that, for specified $c / c_{\mathrm{o}}$ and $k_{\mathrm{o}} H_{\mathrm{o}}$ values, Eq. (4) is easily inverted as a quadratic equation for $k_{\mathrm{o}} h$. When coupled to Eq. (5), however, an iterative method is needed to calculate the depth for each $x_{i}$ location (Newton's iterative method is used).

\subsection{Depth inversion algorithm for $\left(c, L, s_{2} / s_{1}\right)$ data (DIA2)}

As explained above, wave height is not easily obtained from remotely sensed data whereas wave phase is more readily available from current techniques. A modification of DIA1, referred to as DIA2, which eliminates the use of $H$ and only relies on phase information is proposed in the following.

When initially left-right symmetrical waves shoal-up a sloping bottom, due to nonlinearity, they become increasingly asymmetric and skewed (Grilli and Horrillo, 1996, 1997b). Here, asymmetry (defined below) refers to a measure of left-right differences with respect to a crest in a spatial wave profile (Fig. 1b), whereas skewness refers to the distribution of wave elevation $\eta\left(x_{\mathrm{g}}, t\right)$ measured at a given location, say $x=x_{\mathrm{g}}$, about the mean water level. For a $m$ points time series, skewness is thus defined as,

$$
s_{\mathrm{k}}=\frac{\frac{1}{m} \sum_{i=1}^{m}\left(\eta_{i}-\bar{\eta}\right)^{3}}{\left(\frac{1}{m} \sum_{i=1}^{m}\left(\eta_{i}-\bar{\eta}\right)^{2}\right)^{3 / 2}}
$$

with $\bar{\eta}$ the mean elevation at $x=x_{\mathrm{g}}$. In actual depth inversion problems, long time series of wave elevation from which skewness could be calculated are not available whereas spatial variations are (i.e., the 'snapshots'). Hence, wave left-right asymmetry can be spatially quantified in DIAs. In the present applications, asymmetry is defined as the ratio of forward to backward wave slopes $s_{2} / s_{1}$, with $s_{2}(x)=H_{2} / L_{2}$ and $s_{1}(x)=$ $H_{1} / L_{1}$, in which $\left(L_{1}, L_{2}\right)$ and $\left(H_{1}, H_{2}\right)$ denote horizontal and vertical distances from a crest to the previous (i.e., seaward) and next (i.e., landward) troughs, respectively (Fig. 
1b). Since wave height does not change too much over one wavelength, in the applications we further assume, $s_{2} / s_{1} \simeq L_{1} / L_{2}$. Grilli and Horrillo (1996, 1997b) calculated $s_{2} / s_{1}(x)$, for the nine cases in Table 1 (Fig. 6), and showed that this parameter has a high degree of correlation with wave skewness $s_{\mathrm{k}}(x)$.

Now, all that DIA1 really needs as far as wave height information is the deep water wave height $H_{0}$. As a substitute to the wave height data $H\left(x_{i}\right)$ used in DIA1 to retrieve $H_{\mathrm{o}}$, values of $s_{2} / s_{1}\left(x_{i}\right)$ calculated from measured phases (i.e., $L_{1}\left(x_{i}\right)$ and $\left.L_{2}\left(x_{i}\right)\right)$ can be used to estimate $k_{\mathrm{o}} H_{\mathrm{o}}$. To do so, an empirical function of $k h$ and $k_{\mathrm{o}} H_{\mathrm{o}}$ is fitted to the data in Fig. 6. After several trials, the following function of the two parameters was found to give good results,

$$
\begin{aligned}
\frac{s_{2}}{s_{1}}= & \left\{B_{0}+B_{1}(k h)+B_{2}(k h)^{2}+B_{3}(k h)^{3}\right\}+\left\{B_{4}+B_{5}(k h)+B_{6}(k h)^{2}\right. \\
& \left.+B_{7}(k h)^{3}\right\}\left(k_{\mathrm{o}} H_{\mathrm{o}}\right)+\left\{B_{8}+B_{9}(k h)+B_{10}(k h)^{2}+B_{11}(k h)^{3}\right\}\left(k_{\mathrm{o}} H_{\mathrm{o}}\right)^{2} .
\end{aligned}
$$

Values of coefficients $B_{0}$ to $B_{11}$ where found with a least square method, using about 100 multivariate data points per wave case in Table 1 . The coefficient of determination for this curve fit was $R^{2}=96.6 \%$. For reasons explained later, however, in the validation applications for DIA2, it was necessary to repeat this curve fit using results of both the nine cases in Table 1 and the five cases in Table 2. The coefficient of determination for this second curve fit was $R^{2}=95.7 \%$, and the values of coefficients $B_{0}$ to $B_{11}$ where found as, $B_{0}=22.329, B_{1}=-138.01, B_{2}=284.70, B_{3}=-187.90$, $B_{4}=-3.7352, B_{5}=80.763, B_{6}=-2708.3, B_{7}=2260.0, B_{8}=-1228.8, B_{9}=3561.2$, $B_{10}=-670.52$ and $B_{11}=-2907.8$. Fig. 7 shows $s_{2} / s_{1}$ variations calculated based on Eq. (7), using these coefficients, for $k h=0$ to 0.6 and $k_{\mathrm{o}} H_{\mathrm{o}}=0.02$ to 0.14 by steps of 0.02 , as in Fig. 4. This more or less covers the range of variation of $k_{\mathrm{o}} H_{\mathrm{o}}$ in Tables 1 and 2, except for $k_{\mathrm{o}} H_{\mathrm{o}}=0.01$, which is also plotted as curve o on the figure.

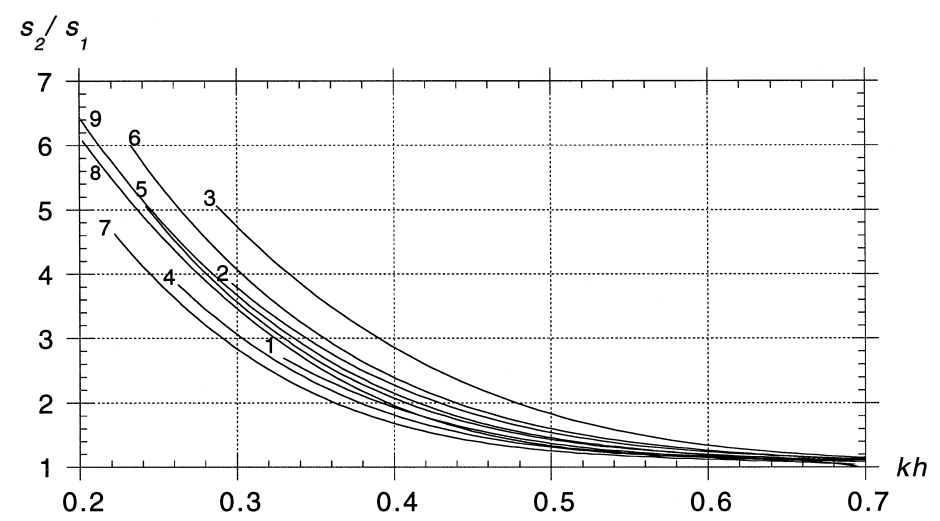

Fig. 6. Wave asymmetry $s_{2} / s_{1}=L_{1} / L_{2}$ (Fig. 1b) computed in the numerical wave tank over a 1:50 slope, for the nine cases listed in Table 1 . Linear theory gives $s_{2} / s_{1}=1$. 


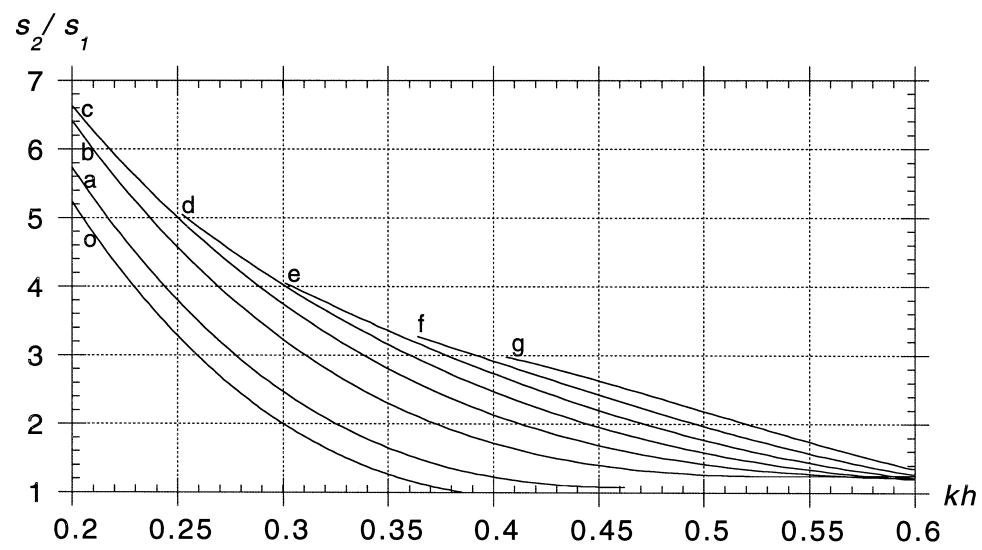

Fig. 7. Empirical curve fit, based on Eq. (7), of wave left-right asymmetry $s_{2} / s_{1}$ computed in numerical wave tank for nine cases in Table 1 and for five cases in Table 2. Curves a to g correspond to $k_{\mathrm{o}} H_{\mathrm{o}}=0.02$ to 0.14 by step 0.02 , as in Fig. 5 , and curve o to $k_{\mathrm{o}} H_{\mathrm{o}}=0.01$. Note, curves have been limited based on $s_{2} / s_{1} \geq 1$ and on the estimation of breaking locations for the steeper waves.

Based on the above, the layout for DIA2 is as follows $(i=1, \ldots, N)$.

(1) The period $T$ (and thus $k_{\mathrm{o}}$ ) is first predicted, as for DIA1, using $L_{\mathrm{c}}\left(x_{i}\right), L_{\mathrm{t}}\left(x_{i}\right)$ and $c\left(x_{i}\right)$ values.

(2) A first guess is made for $H_{\mathrm{o}}$, say $H_{\mathrm{o} 1}$, as a small but finite value, and a first depth variation $h_{1}\left(x_{i}\right)$ is calculated for this guess as in DIA1 (i.e., for $k_{\mathrm{o}} H_{\mathrm{o} 1}$ ), using the inverted Eqs. (4) and (5) and the $c\left(x_{i}\right)$ values.

(3) Based on $h_{1}\left(x_{i}\right), k h_{1}\left(x_{i}\right)=k_{\mathrm{o}} h_{1}\left(x_{i}\right) /\left(c\left(x_{i}\right) / c_{\mathrm{o}}\right)$ is obtained, and a new corrected value of $H_{\mathrm{o}}$, say $H_{\mathrm{o} 2}$, is predicted using $s_{2} / s_{1}\left(x_{i}\right)$ values, by inverting Eq. (7) for each $x_{i}$, as a quadratic equation for $k_{\mathrm{o}} H_{\mathrm{o} 2}$, and taking the mean of all such predictions.

(4) Steps (2) and (3) are iteratively repeated, for $n=2,3, \ldots$, until convergence is reached on $H_{\mathrm{o} n}$ and $h_{n}(x)$ according to some preset criteria.

\section{Numerical validation of depth inversion algorithms}

\subsection{Depth inversion algorithm for $(c, H, L)$ data (DIA1)}

Figs. 8 and 10 show five validation tests of the application of DIA1 to the depth inversion problem. In each case, direct computations are first made in the numerical wave tank, using the corresponding bottom topography, for the shoaling of an incident streamfunction wave with height $H_{\mathrm{o}}$ and period $T$ at depth $h_{\mathrm{o}}$, and a data set of simultaneous $\left(x, c, H, L_{\mathrm{c}}, L_{\mathrm{t}}\right)$ values is numerically generated. DIA1 is applied to this data set and a prediction of the bottom topography $h(x)$ is made, which is then compared to the actual topography. The accuracy of the depth prediction is finally 

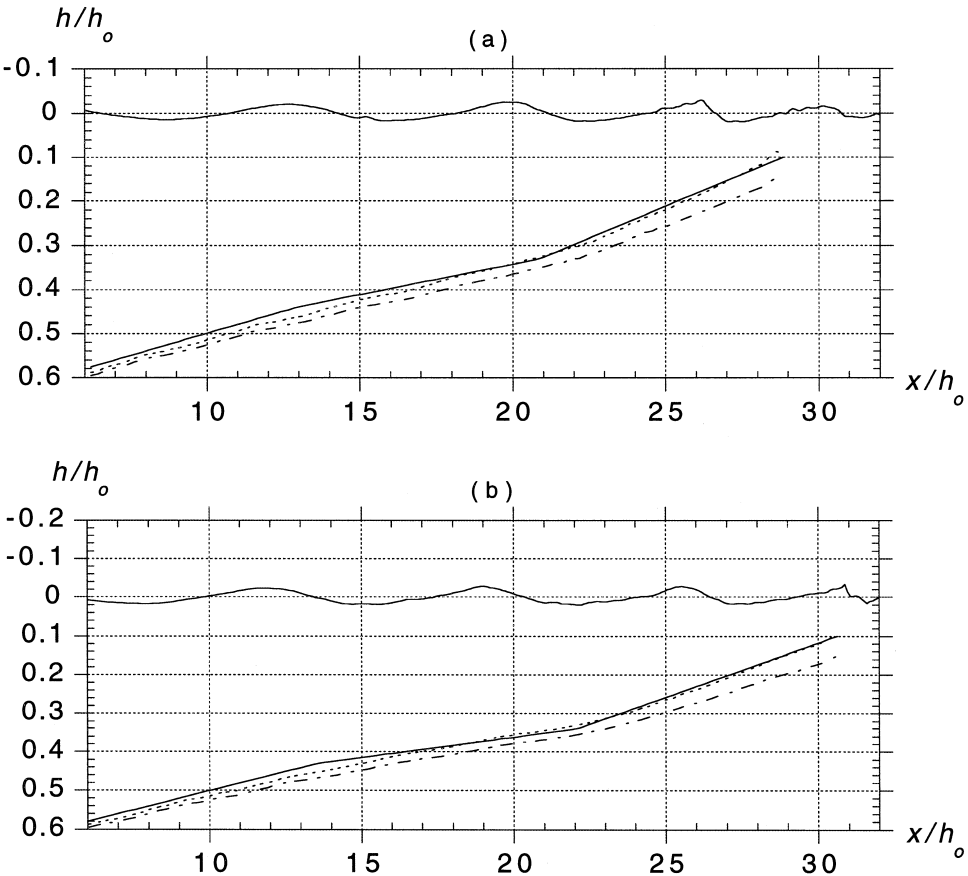

Fig. 8. Depth inversion cases: (a) b1 (broken slope 1:50, 1:70, 1:35) with $\eta(x)$ at $t^{\prime}=146.3=12.8 T^{\prime}$; (b) b2 (broken slope $1: 50,1: 100,1: 35)$ with $\eta(x)$ at $t^{\prime}=156.3=13.6 T^{\prime}$. (— True bottom topography $h(x)$; (----) estimated topography with DIA1 (note: $h(x)$ estimates are found closely similar with DIA2); (-- - ) estimated topography with linear dispersion relation. $h_{\mathrm{o}}$ is a reference depth scale. Note: bottom is plotted up to the entrance of the absorbing beach in the numerical wave tank (Fig. 1).

assessed in terms of both root-mean-square (rms) and maximum errors. Note, for $N$ values $y_{i}$ of a variable $y$ at points $x_{i}$, and $N$ predictions $\tilde{y}_{i}$, the rms-error is defined as,

$$
\operatorname{rms}(y)=\left\{\frac{1}{N} \sum_{i=1}^{N}\left(\frac{y_{i}-\tilde{y}_{i}}{y_{i}}\right)^{2}\right\}^{1 / 2} .
$$

Table 2 gives general data and results for these cases.

\subsubsection{Broken slopes}

For the two cases in Fig. 8, the bottom is made of a succession of three slopes (which is somewhat similar to the natural beach profile sketched in Fig. 1): (a) 1:50, 1:70, and 1:35 (case b1); or (b) 1:50, 1:100, and 1:35 (case b2). Shoaling of a long incident periodic wave with period $T^{\prime}=11.47$ and height $H_{\mathrm{o}}^{\prime}=0.035$ is computed in both cases. Fig. $8 \mathrm{a}$ and $\mathrm{b}$ (upper parts) show examples of computed free surface elevations $\eta(x)$ and we can see that incident waves are fairly long with respect to depth and that many smaller scale oscillations are being generated on the waves as they shoal up in the shallower water region, indicating the transfer of energy to higher-order harmonics resulting from increasing nonlinearity. (No attempts were made in these computations to 
more accurately resolve these small scale oscillations.) Selected results of these computations, i.e., about $N=100$ values of $\left(c, H, L_{c}, L_{\mathrm{t}}\right)$ as a function of $x$, taken between depth $0.6 h_{\mathrm{o}}$ and $0.1 h_{\mathrm{o}}$, were used in DIA1. Due to the low incident wave steepness $k_{\mathrm{o}} H_{\mathrm{o}}=0.0105<\gamma_{\mathrm{oa}}$, the interpolation defined by Eq. (5) was used in the calculations, together with the nonlinear celerity Eq. (4). The wave period was first predicted, as explained above, using the computed values of $c, L_{\mathrm{c}}$, and $L_{\mathrm{t}}$; predicted periods are given in Table 2 as $T_{\mathrm{p}}^{\prime}$ and are found to be within 0.7 and $0.3 \%$ of the true period, for cases b1 and b2, respectively. Deep water wave heights and bottom depth variations were then predicted iteratively for each case, using the computed $c$ and $H$ values and $T_{\mathrm{p}}^{\prime}$. Deep water wave heights are given in Table 2 as $H_{\mathrm{po}}^{\prime}$ and are found to be within 0.8 and $0.7 \%$ of the true height, for cases b1 and b2, respectively.

To assess the validity of applying results of computations on a 1:50 slope to these more complex bottom topographies, Fig. 9 shows comparisons between the values of $c$, $H$, and $h$, computed as a function of $k h$, and their predictions with both DIA 1 and linear wave theory. Note that cases b1 and b2 have a significant part of their results in the shallow water region $(k h<\pi / 10)$. For the direct prediction of $c$, despite the use of interpolation Eq. (5), the present algorithm gives small rms-errors, $\mathrm{rms}_{c}$, of 1.2 and $1.1 \%$ for cases b1 and b2, respectively (Table 2). (Note that, despite the different (mildly varying) bottom topography, due to the same $k_{\mathrm{o}} H_{\mathrm{o}}$ value for cases b1 and b2, as expected from Grilli and Horrillo's conclusions, both calculated $c$ and $H$ variations are

(a)

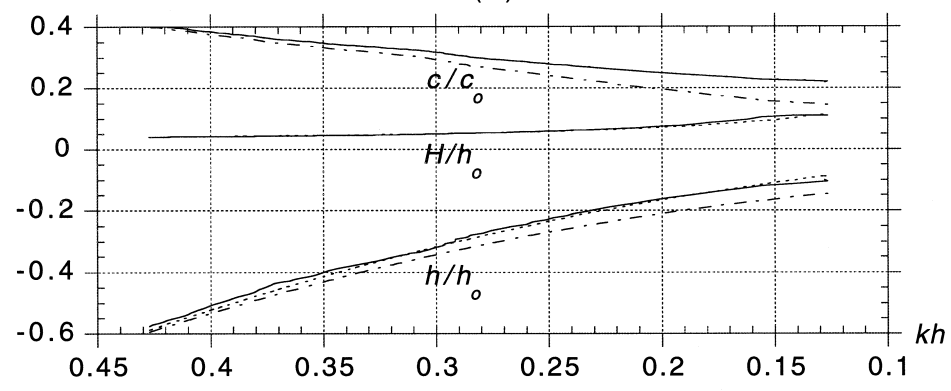

(b)

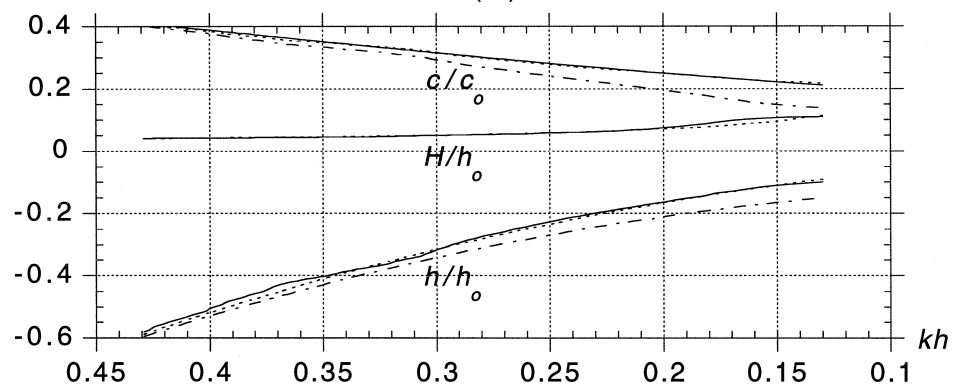

Fig. 9. Depth inversion cases: (a) b1; (b) b2. (— estimated values with DIA1 (note: estimates of $c / c_{\mathrm{o}}$ and $h / h_{\mathrm{o}}$ are found closely similar with DIA2); (- -) estimated values with linear dispersion relation. 
closely identical in Fig. 9a and b, when plotted as a function of $k h$.) In each case, wave celerity is underpredicted by linear wave theory, particularly for $k h<0.35$. For the final prediction of $H$, after iterations have converged in DIA1, the algorithm gives $\mathrm{rms}_{H}$ errors of 2.8 and $3.8 \%$ for cases b1 and b2, respectively (Table 2).

Fig. 8 (bottom parts) gives the comparison, as a function of $x$, between the true bottom depth $h(x)$, the depth predicted using DIA1, and the depth predicted using the linear dispersion relation. Clearly, DIA1 provides a much closer prediction of the bottom topography than when using the linear dispersion relation. This is further illustrated in Fig. 12a which shows, for each case, the relative error between the nonlinear or the
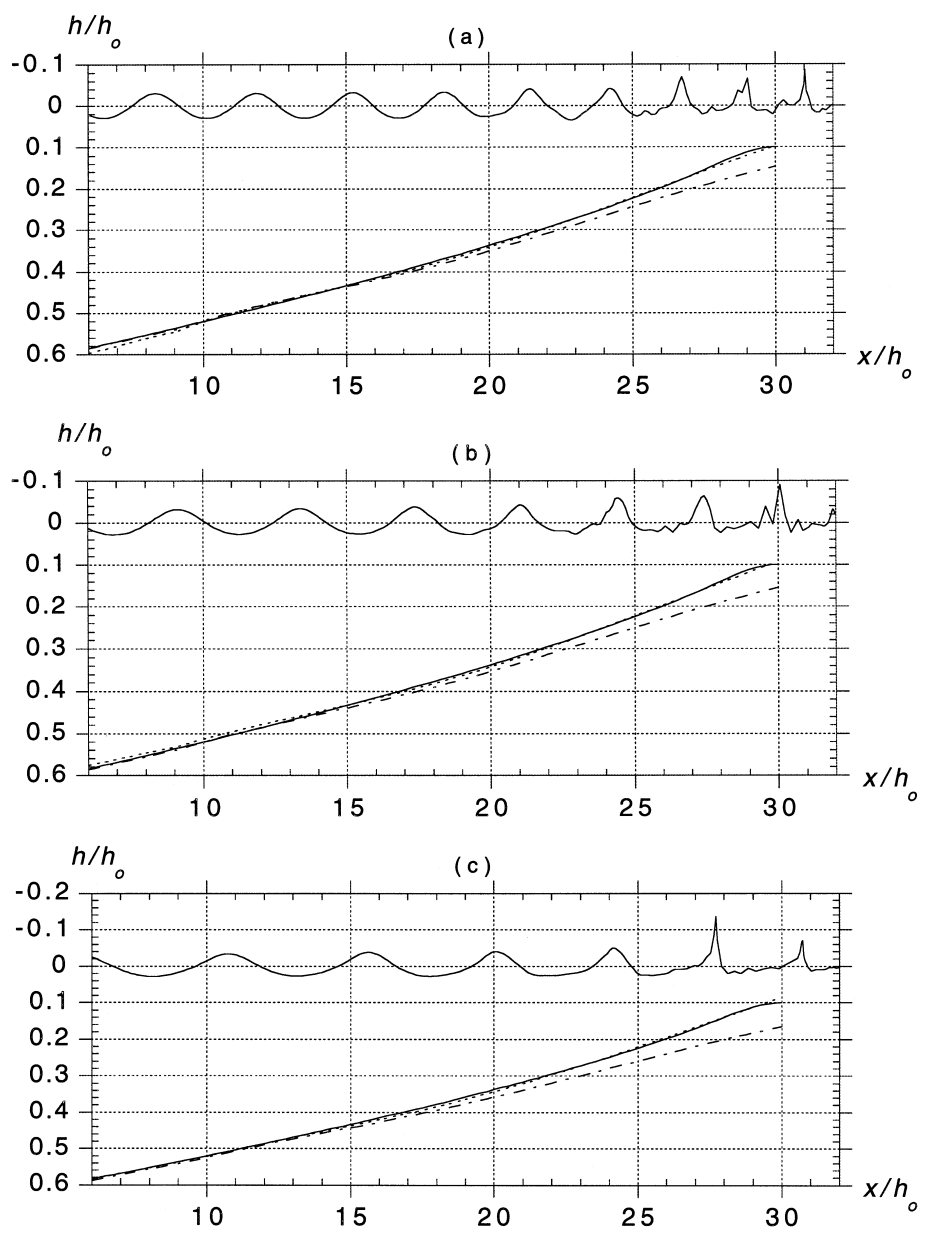

Fig. 10. Depth inversion for an equilibrium beach with average slope 1:50, cases: (a) $\mathrm{n} 1$ with $\eta(x)$ at $t^{\prime}=112.8=20.5 T^{\prime}$; (b) n2 with $\eta(x)$ at $t^{\prime}=112.1=17.3 T^{\prime}$; (c) n3 with $\eta(x)$ at $t^{\prime}=106.9=14.3 T^{\prime}$. (- True bottom topography $h(x)$; (----) estimated topography with DIA1 (note: $h(x)$ estimates are found closely similar with DIA2); (- - ) estimated topography with linear dispersion relation. Note: bottom is plotted up to the entrance of the absorbing beach in the numerical wave tank (Fig. 1). 
linear predictions of depth, and the actual depth $h_{i}$ at points $x_{i}$. Corresponding rms and maximum errors are given in Table 2. Errors of the DIA are only a few percent for most of the wave propagation distance (rms-errors are 3.6 and $2.7 \%$, respectively), whereas errors of the method based on linear dispersion are always larger and reach very large values (order 50\%), in the shallower water region, particularly for $k h<\pi / 10$.

\subsubsection{Natural slopes}

According to the theory of Dean (1991), in average, natural beaches tend to follow a bottom depth variation defined as, $h=A\left(x^{*}-x\right)^{2 / 3}$, with $x^{*}$ denoting the location of depth $h_{\mathrm{o}}^{*}$ at the toe of the slope, and $A$ a constant depending on the specified average slope (which actually is related to the mean sediment grain size). In Fig. 1, the measured

(a)

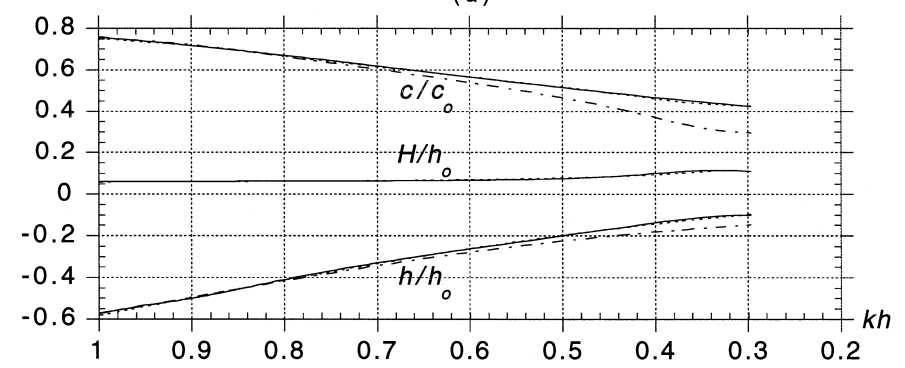

(b)

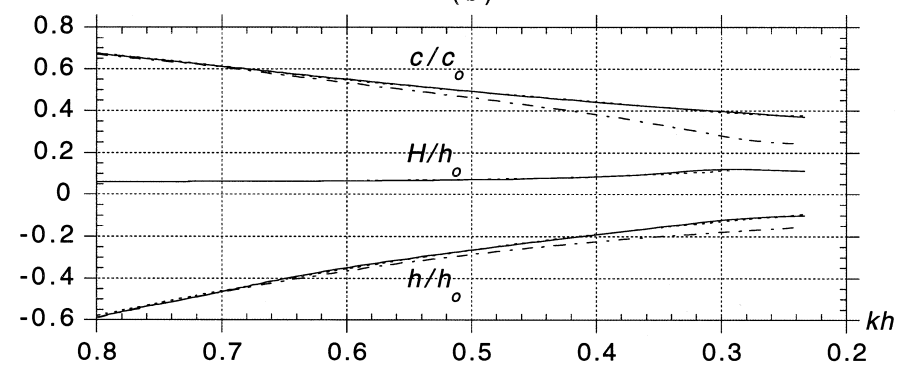

(c)

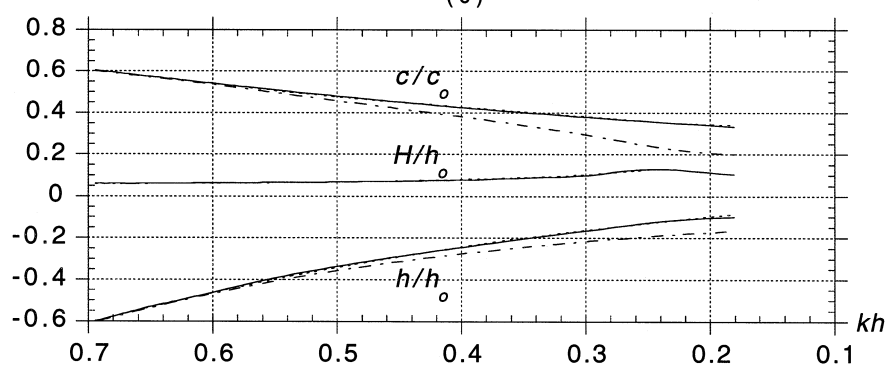

Fig. 11. Depth inversion cases: (a) n1; (b) n2; (c) n3. (—) True $h$, and computed $H / H_{\mathrm{o}}, c / c_{\mathrm{o}}$; (----) estimated values with DIA1 (note: estimates of $c / c_{\mathrm{o}}$ and $h / h_{\mathrm{o}}$ are found closely similar with DIA2); ( - - - estimated values with linear dispersion relation. 
bottom topography at Ft. Walton Beach, FL, was sketched as an example, and an average equilibrium shape was fitted as $h / h_{\mathrm{o}}=0.0758\left(74.20-x / h_{\mathrm{o}}\right)^{0.64}$; hence, 0.64 $\simeq 2 / 3$. The curve fit had a $R^{2}=0.98$, which indicates a good overall agreement. As can be seen on Fig. 1 (chained line), the equilibrium beach profile has a milder slope in deeper water and a steeper slope in shallower water, than the average value, 1:82 in this case.

Three shoaling cases, referred to as n1, n2, and, n3 in Table 2, were computed for the same bottom topography in the numerical wave tank, consisting in Dean's equilibrium beach profile with an average slope 1:50, using three different incident waves of height $H_{\mathrm{o}}^{\prime *}=0.06$ and periods $T^{\prime}=5.5,6.5$, and 7.5, respectively, resulting in waves of decreasing incident steepness; Fig. 10a-c show examples of free surface elevations $\eta(x)$ calculated for these waves (upper parts). Selected results of these computations, i.e., about $N=100$ values of $\left(c, H, L_{c}, L_{\mathrm{t}}\right)$ as a function of $x$, again taken between depth $0.6 h_{\mathrm{o}}$ and $0.1 h_{\mathrm{o}}$, were used in DIA1. Due to the larger incident wave steepness for these cases, $k_{\mathrm{o}} H_{\mathrm{o}}=0.0846,0.0591,0.0430$, the interpolation defined by Eq. (5) was not used in the calculations but only the nonlinear celerity Eq. (4). Predicted wave periods $T_{\mathrm{p}}^{\prime}$ were found to be within $0.4,0.3$, and $0.1 \%$ of the true period, and deep water wave heights $H_{\mathrm{po}}^{\prime}$ within $1.1,1.7$, and $1.9 \%$ of the true height, for cases n1, n2 and n3, respectively (Table 2 ).

As for the broken slope cases, Fig. 11 gives simultaneous comparisons between the values of $c, H$, and $h$, computed as a function of $k h$, and their predictions with DIA1
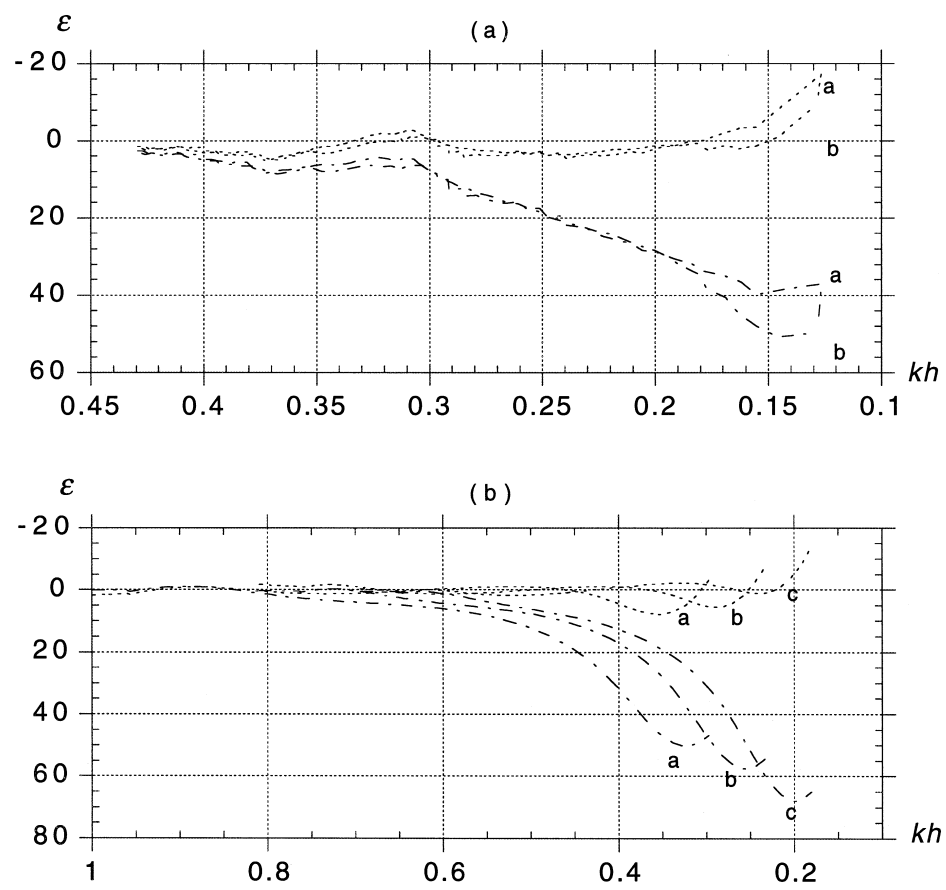

Fig. 12. Relative errors, $\varepsilon=\left(h_{i}-\tilde{h}_{i}\right) / h_{i}$, for five cases in Table 2: [(a) a: b1, b: b2; (b) a: n1, b: n2, c: n3] for depth prediction $\tilde{h}_{i}$ at $x_{i}$ with: (----) DIA1; ( - - $)$ linear dispersion relation. 
and linear wave theory. These waves were barely entering shallow water at the upper part of the slope. For the direct prediction of $c$, DIA1 gives very small $\mathrm{rms}_{c}$ errors of $0.6,0.7$, and $0.4 \%$ for cases $\mathrm{n} 1, \mathrm{n} 2$ and $\mathrm{n} 3$, respectively (Table 2 ). All the $k_{\mathrm{o}} H_{\mathrm{o}}$ values for these cases are in the range of the original computations based on which Eq. (4) was derived; the bottom topography, however, is different. The low rms values, hence, further confirm the hypothesis at the basis of our DIAs that celerity can be predicted for a variety of mildly sloping topographies based on results obtained for a simple plane slope. As for cases b1 and b2, in each case, wave celerity is underpredicted by linear wave theory, particularly for $k h<0.60$. For the final prediction of $H$, after iterations have converged in DIA1, the algorithm gives $\mathrm{rms}_{H}$ errors of 2.7, 2.0 and $2.0 \%$ for cases $\mathrm{n} 1, \mathrm{n} 2$ and $\mathrm{n} 3$, respectively (Table 2).

Fig. 10 (bottom part) gives the comparison, as a function of $x$, between the true bottom depth $h(x)$, the depth predicted using DIA1, and the depth predicted using the linear dispersion relation. Again, it is clear that DIA1 provides a much closer prediction of the bottom topography than when using the linear dispersion relation. This is further illustrated in Fig. 12b which shows, for each case, the relative error between the nonlinear and linear predictions of depth, and the actual depth, respectively. Values of rms and maximum errors are also given in Table 2. Errors of the DIA are only a few percent for most of the wave propagation distance (rms-errors are 2.1, 1.7 and 2.0\%, respectively), whereas errors of the method based on linear dispersion are always larger and reach very large values (order 50-70\%), particularly, in the shallower water region for $k h<0.60$. Finally, it should also be pointed out that the single bottom topography for cases $n 1, n 2$, and $n 3$ was predicted with the same level of accuracy (rms-error) for the three different incident waves. This indicates that in actual field situations, results of the DIA obtained for several incident waves, with different characteristics, can be averaged to obtain a better prediction of the depth variation.

\subsection{Depth inversion algorithm for $\left(c, L, s_{2} / s_{1}\right)$ data (DIA2)}

Depth inversion for the five test cases of Table 2, used to validate DIA1, was repeated using DIA2. For the natural slope cases n1, n2, n3, small rms-errors of 2.1, 3.4, and $3.2 \%$, respectively, were readily obtained for the depth prediction, using the curve fit of Eq. (7) to the data from the nine cases in Table 1 to estimate $H_{\mathrm{o}}$. Results in Fig. 11 for $c / c_{\mathrm{o}}$ and $h / h_{\mathrm{o}}$ were also closely reproduced using DIA2. Errors on the direct prediction of $H(x)$, however, were larger than with DIA1 $\left(\mathrm{rms}_{H}=2.7,13.4\right.$, and $13.1 \%$, respectively), indicating that detailed information on wave height is not so important, provided $H_{\mathrm{o}}$ is correctly estimated. These good results confirmed that DIA2 is also a viable depth inversion method. For the broken slope cases b1 and b2, rms-errors on depth were initially much larger, at 19.2 and $20.9 \%$, respectively. This was later explained by the fact that none of the calibration computations in Table 1 were performed for a small enough value of $k_{\mathrm{o}} H_{\mathrm{o}}$, whereas $k_{\mathrm{o}} H_{\mathrm{o}}=0.01$ in cases b1 and b2. For the celerity, the interpolation Eq. (5) provided accurate results for cases b1 and b2. The initial empirical fit of $s_{2} / s_{1}$ for the cases in Table 1 , however, did not cover the range of small $k_{\mathrm{o}} H_{\mathrm{o}}$ values. The curve fit of $s_{2} / s_{1}$ values with Eq. (7) was thus 
repeated, including all the cases in Table 2 in addition to those in Table 1. Coefficients $B_{i}$ 's slightly changed but a good $R^{2}=95.7 \%$ was still obtained (note, these changes did not affect the accuracy of results for cases $\mathrm{n} 1, \mathrm{n} 2$, and $\mathrm{n} 3$ ). Using the new empirical equation for $s_{2} / s_{1}$ in DIA2, the algorithm was re-applied to cases b1 and b2 and rms-errors on depth were significantly reduced to 5.7 and $3.9 \%$, respectively. For these cases, using DIA2, detailed results in Fig. 9 for $c / c_{\mathrm{o}}$ and $h / h_{\mathrm{o}}$ were also well reproduced; again, larger errors occurred on the direct $H$ prediction than when using DIA1 $\left(\mathrm{rms}_{H}=11.4\right.$ and $10.3 \%$, respectively).

\section{Conclusions}

In this paper, two DIAs were developed and validated based on results of computations for the shoaling of periodic waves over mild slopes, in a two-dimensional numerical wave tank based on FNPF theory (Grilli and Horrillo, 1996, 1997b). In actual field situations, the first algorithm, DIA1, uses sets of values of wave celerity $c$, height $H$, and spatial wavelengths $L_{\mathrm{c}}$ and $L_{\mathrm{t}}$, simultaneously measured at a number of locations $x_{i}(i=1, \ldots, N)$ in the direction of wave propagation, e.g., using video or radar remote sensing techniques, to predict the depth variation $h\left(x_{i}\right)$. The second algorithm, DIA2, uses spatial wave asymmetry $s_{2} / s_{1}$ calculated from wave phase, instead of wave height information.

In the DIAs, a bi-quadratic empirical relationship is first derived to express $c$ as a function of relative depth $k_{\mathrm{o}} h$ and deep water steepness $k_{\mathrm{o}} H_{\mathrm{o}}$, based on results of FNPF computations for nine cases of periodic waves shoaling over a 1:50 slopes, with $k_{\mathrm{o}} H_{\mathrm{o}}=0.029-0.114$ and $k_{\mathrm{o}} h \leq 0.5$. This relationship has a $R^{2}=99.8 \%$ coefficient of determination. An extension of this relationship for smaller wave steepness is proposed, using an interpolation with results of linear wave theory.

To carry out depth inversion, wave period is first predicted based on the mean of observed $L / c$ values, where $L$ is found as the average of $L_{\mathrm{c}}$ and $L_{\mathrm{t}}$, and $H_{\mathrm{o}}$ is found: (i) in DIA1, based on the observed $H$ values, using the wave steepness variation from linear wave theory and the nonlinear celerity; (ii) in DIA2, based on an empirical fit of wave asymmetry values $s_{2} / s_{1}$, calculated in the numerical wave tank, as a function of $k h$ and $k_{\mathrm{o}} H_{\mathrm{o}}\left(R^{2}=96 \%\right)$. Due to the dependence of $c$ on $H_{\mathrm{o}}$, an iterative method is needed in both algorithms. The celerity relationship is then inverted to predict depth $h$.

The DIAs were validated by applying them to results of computations for five cases with more complex bottom topographies and different incident waves than in the original computations. In all cases, rms-errors for the depth predictions were found to be less than a few percent (1.7-3.6\% for DIA1; $2.1-5.7 \%$ for DIA2), whereas depth predictions based on the linear dispersion relation gave rms-errors 5 to 10 times larger (14.6-20.5\%). The direct predictions of $c$ and $H$ were also found to be accurate within a few percent, particularly for DIA1. This indicates that both DIAs are applicable to realistic mildly and monotonously sloping bottom topographies. Overall, although rms-errors on depth prediction are slightly larger with DIA2 than with DIA1, they are still significantly smaller than those corresponding to linear wave theory (three to seven times smaller). This clearly confirms the relevance of DIA2's approach for carrying out 
depth inversion. Since it is purely based on parameters derived from wave phase, DIA2 is more easily applicable to field data than DIA1.

Results also indicate that depth inversion methods solely based on the linear dispersion relation may lead to large errors (50-70\%) for the depth prediction in very shallow water, where wave nonlinearity and asymmetry are large and amplitude dispersion effects cannot be neglected in the celerity relationship.

Besides being based on a two-dimensional method, the main limitations of the proposed DIAs, and hence sources of errors in depth prediction, are the use of periodic incident waves and topographies with monotonously decreasing and mildly varying depth. Computations could be run in the numerical wave tank, however, to assess how incident wave groups with fairly narrow band could be used in the DIAs, instead of purely periodic waves. Also variations of $c, H$, and $s_{2} / s_{1}$ for waves propagating over shallow bars, i.e., with a decreasing depth onshore of the bar's crest, could be studied in the numerical wave tank and included in the DIAs (see the work of Grilli and Horrillo (1998) for preliminary results for barred-beaches). Finally, nothing prevents the present approach to be extended to three dimensions, although a three-dimensional FNPF numerical wave tank will be computationally very expensive. These studies will be the object of continuations of this work.

\section{Acknowledgements}

This publication is the result of research sponsored by the US Naval Research Laboratory, Stennis Space Center, grants N-00014-95-1-G607 and N-00014-96-C012, from the Remote Sensing Division (code 7240). The kind assistance of Dr. Peter Smith from NRL-SSC and Dr. John Dugan from Areté Associates is gratefully acknowledged. Anonymous reviewers are thanked for their helpful suggestions for improving the manuscript.

\section{References}

Dean, R.G., 1991. Equilibrium beach profiles: characteristics and applications. J. Coastal Res. 7 (1), 53-84. Dean, R.G., Dalrymple, R.A., 1984. Water Wave Mechanics for Engineers and Scientists. Prentice-Hall, Englewood Cliffs, NJ.

Dommermuth, D.G., Yue, D.K.P., Lin, W.M., Rapp, R.J., Chan, E.S., Melville, W.K., 1988. Deep-water plunging breakers: a comparison between potential theory and experiments. J. Fluid Mech. 189, 423-442.

Dugan, J., 1997. Bathymetry measurements from long range airborne imaging systems. Proc. 4th Int. Conf. on Remote Sensing for Marine and Coastal Environments. Orlando, FL.

Dugan, J., Forsyth, C., Suzukawa, H., Farber, M., 1996. Ocean wave dispersion surface measured with airborne IR imaging system. IEEE Trans. Geosci. Remote Sensing 34 (4), 182-1284.

Grilli, S.T., Horrillo, J., 1996. Fully nonlinear properties of periodic waves shoaling over slopes. Proc. 25th Int. Conf. on Coastal Engineering, Orlando, USA. ASCE edn., Vol. 1, pp. 717-730.

Grilli, S.T., Horrillo, J., 1997a. Numerical generation and absorption of fully nonlinear periodic waves. J. Eng. Mech. 123 (10), 1060-1069.

Grilli, S.T., Horrillo, J., 1997b. Computation of properties of periodic waves shoaling over mild slopes in a fully nonlinear numerical wave tank. J. Geophys. Res., submitted. 
Grilli, S.T., Horrillo, J., 1998. Periodic waves shoaling over barred-beaches in a fully nonlinear numerical wave tank. Proc. 8th Int. Offshore and Polar Eng. Conf. Montréal, Canada, Int. Soc. Offshore and Polar Engineering ed., Vol. 3, pp. 299-300.

Grilli, S.T., Subramanya, R., 1996. Numerical modeling of wave breaking induced by fixed or moving boundaries. Comput. Mech. 17, 374-391.

Grilli, S.T., Skourup, J., Svendsen, I.A., 1989. An efficient boundary element method for nonlinear water waves. Eng. Anal. Boundary Elements 6 (2), 97-107.

Grilli, S.T., Subramanya, R., Svendsen, I.A., Veeramony, J., 1994. Shoaling of solitary waves on plane beaches. J. Waterway Port Coastal Ocean Eng. 120 (6), 609-628.

Grilli, S.T., Svendsen, I.A., Subramanya, R., 1997. Breaking criterion and characteristics for solitary waves on slopes. J. Waterway Port Coastal Ocean Eng. 123 (3), 102-112.

Lippmann, T.C., Holman, R.A., 1989. Quantification of sand bar morphology: a video technique based on wave dissipation. J. Geophys. Res. 94 (C1), 995-1011.

Lippmann, T.C., Holman, R.A., 1990. The spatial and temporal variability of sand bar morphology. J. Geophys. Res. 95 (C7), 11575-11590.

Lippmann, T.C., Holman, R.A., 1991. Phase speed and angle of breaking waves measured with video techniques. Proc. Coastal Sediment 1991 Conf. ASCE edn., pp. 542-556.

Lippmann, T.C., Holman, R.A., 1992. Wave group modulations in cross-shore breaking patterns. Proc. 23rd Int. Conf. on Coastal Engineering, Venice, Italy. ASCE edn., Vol. 1, pp. 918-931.

Madsen, P.A., Murray, R., Sorensen, O.R., 1991. A new form of the Boussinesq equations for nearshore wave propagation. Coastal Eng. 15, 371-388.

Nwogu, O., 1993. An alternative form of the Boussinesq equations for nearshore wave propagation. J. Waterway Port Coastal Ocean Eng. 119 (6), 618-638.

Ohyama, T., Beji, S., Nadaoka, K., Battjes, J.A., 1994. Experimental verification of numerical model for nonlinear wave evolution. J. Waterway Port Coastal Ocean Eng. 120 (6), 637-644.

Sobey, R.J., Bando, K., 1991. Variations on higher-order shoaling. J. Waterway Port Coastal Ocean Eng. 117 (4), 348-368.

Svendsen, I.A., Jonsson, I.G., 1980. Hydrodynamics of coastal regions. Technical University of Denmark Pub.

Tsai, W.T., Yue, D.K.P., 1996. Computation of nonlinear free-surface flows. Annu. Rev. Fluid Mech. 28, $249-278$.

Wei, J., Kirby, J.T., Grilli, S.T., Subramanya, R., 1995. A fully nonlinear Boussinesq model for surface waves: I. Highly nonlinear unsteady waves. J. Fluid Mech. 294, 71-92.

Williams, J.Z., Dugan, J., 1997. Bathymetry measurements using electro-optical remote sensing. Proc. 4th Int. Conf. on Remote Sensing for Marine and Coastal Environments, Orlando, FL. 